Mr A. S. Ramsey kindly drew my attention to a flaw in the proof which I gave in my Differential Geometry, Vol. 1, p. 22. This may be rectified by considering $S$ as the limiting position of a point which is equidistant from the four points $P, P_{1}, P_{2}, P_{3}$ as these tend to coincidence at $P$. In other words $S$ is the limiting position of the intersection of four equal spheres with centres at these four points. Let $R$ be their common radius. Then, if $\mathrm{s}$ is the position vector of the current point on the sphere whose centre is at $P$, the equation of the sphere is

$$
(\mathrm{s}-\mathrm{r})^{2}=R^{2}
$$

The position of the ultimate point of intersection of the spheres is then found from the three equations obtained by three differentiations of (5) with respect to $s$.

\title{
The advantage of differentials in the technique of differentiation
}

By E. G. Phillips, University College of North Wales, Bangor.

In the last few years the topic of differentials has occupied the attention of a good many teachers of mathematics. Since the appearance in 1931 of $\mathrm{my}$ article ${ }^{1}$ advocating the teaching of the Differential Calculus from the differential standpoint right from the start, very divergent views have been expressed on this question and there was a general discussion on the topic at the annual meeting of the Mathematical Association in 1934. This article, the subsequent correspondence and the discussion have certainly brought into evidence the fact that many mathematicians still have a very obvious distrust of "differentials" and they seem unable to clear their minds of some early prejudice against the employment of differentials for any purpose.

The advantages of the differential notation are most evident in the technique of differentiation of functions of more than one variable. The superiority of the method of differentials over the

1 Math. Gasette XV (1931), 401. 
more common method which uses partial derivatives may be very clearly seen by contrasting the two solutions of the following problem:-

The three variables $x, y, z$ are connected by a functional relation $F(x, y, z)=0:$ prove that

$$
\left(\frac{\partial x}{\partial z}\right)^{-3} \frac{\partial^{2} x}{\partial y \partial z}=\frac{\partial z}{\partial y} \frac{\partial^{2} z}{\partial x^{2}}-\frac{\partial z}{\partial x} \frac{\partial^{2} z}{\partial x \partial y}
$$

where on the right-hand side the independent variables are $x, y$ and on the left-hand side they are $y, z$.

In the customary notation we write

$$
p=\frac{\partial z}{\partial x}, \quad q=\frac{\partial z}{\partial y}, \quad r=\frac{\partial^{2} z}{\partial x^{2}}, \quad s=\frac{\partial^{2} z}{\partial x \partial y}, \quad t=\frac{\partial^{2} z}{\partial y^{2}} .
$$

Solution 1. By differentials.

When $x$ and $y$ are the independent variables, $z$ is a function of $x$ and $y$ satisfying $F(x, y, z)=0$ and so

$$
d z=p d x+q d y \text {. }
$$

Now equation ( 1 ) is permanent, whatever the independent variables may be, and so we may differentiate it again, but if $y$ and $z$ are the independent variables $d y$ and $d z$ are constants, hence

$$
0=r d x^{2}+2 s d x d y+t d y^{2}+p d^{2} x
$$

All that remains is to express $d^{2} x$ in terms of the differentials of the independent variables $d y$ and $d z$ and use (1) to eliminate the $d x$ in (2): we can rewrite (1) in the form

and, from (2),

$$
d x=\frac{1}{p} d z-\frac{q}{p} d y
$$

$$
d^{2} x=-\frac{1}{p^{3}}\left\{r d z^{2}+2(p s-q r) d y d z+\left(q^{2} r-2 p q s+p^{2} t\right) d y^{2}\right\}
$$

and, on selecting the coefficient of $2 d y d z$ we get

From (3) we get

$$
\frac{\partial^{2} x}{\partial y \hat{c z}}=-\frac{p s-q r}{p^{3}}
$$

and the required result follows,

$$
\frac{\partial x}{\partial z}=\frac{1}{p}
$$

$$
\left(\frac{\partial x}{\partial z}\right)^{-3} \frac{\partial^{2} x}{\partial y \partial z}=q r-p s
$$


siv

Solution 2. By partial derivatives.

In this method we have to keep in mind which are the independent variables at every step: even the notation required is far more complicated than in Solution 1.

We have $F(x, y, z)=0$ and so

$$
\frac{\partial F}{\partial x} d x+\frac{\partial F}{\partial y} d y+\frac{\partial F}{\partial z} d z=0,
$$

and when $x$ and $y$ are the independent variables

$$
\frac{\partial z}{\partial x}=-\frac{F_{x}}{F_{z}}, \quad \frac{\partial z}{\partial y}=-\frac{F_{y}}{F_{z}} .
$$

If $y$ and $z$ are the independent variables we get similarly

Now

$$
\frac{\partial x}{\partial y}=-\frac{F_{y}}{F_{x}}, \quad \frac{\partial x}{\partial z}=-\frac{F_{z}}{F_{x}} .
$$

and so

$$
\begin{aligned}
\frac{\partial^{2} x}{\partial y \partial z} & =\left(\frac{\partial}{\partial y}\right)_{z}\left(\frac{\partial x}{\partial z}\right)=-\left(\frac{\partial}{\partial y}\right)_{z}\left(\frac{F_{z}}{F_{x}}\right) \\
& =-\frac{1}{\boldsymbol{F}_{x}^{2}}\left\{\boldsymbol{F}_{x}\left(\boldsymbol{F}_{z y}+\boldsymbol{F}_{z x} \frac{\partial x}{\partial y}\right)-\boldsymbol{F}_{z}\left(\boldsymbol{F}_{x y}+\boldsymbol{F}_{x x} \frac{\partial x}{\partial y}\right)\right\}
\end{aligned}
$$

$$
\begin{aligned}
\left(\frac{\partial x}{\partial z}\right)^{-3} \frac{\partial^{2} x}{\partial y \partial z} & =-\frac{F_{x}}{F_{z}^{3}}\left\{F_{x} F_{z y}-F_{z} F_{x y}+\left(F_{x} F_{z x}-F_{z} F_{x x}\right) \frac{\partial x}{\partial y}\right\} \\
& =-\frac{F_{x}}{F_{z}} \frac{F_{x} F_{z y}-F_{z} F_{x y}}{F_{z}^{2}}-\frac{F_{x} F_{z x}-F_{z} F_{x x}}{F_{z}^{2}} \frac{F_{x}}{F_{z}}\left(-\frac{F_{y}}{F_{x}}\right) \\
& =\frac{\partial z}{\partial x} \frac{F_{x} F_{z y}-F_{z} F_{x y}}{F_{z}^{2}}-\frac{F_{x} F_{z x}-F_{z} F_{x x}}{F_{z}^{2}} \frac{\partial z}{\partial y} \\
& =\frac{\partial z}{\partial x}\left\{\left(\frac{\partial}{\partial y}\right)_{x}\left(\frac{F_{x}}{F_{z}}\right)-\frac{\left.F_{x z} F_{z} \frac{\partial z}{\partial y}-F_{z z} F_{x} \frac{\partial z}{\partial y}\right\}}{F_{z}^{2}}\right\} \\
& \left.-\frac{\partial z}{\partial x}\left(\frac{\partial}{\partial y}\right)_{x}\left(\frac{F_{x}}{F_{z}}\right)-\frac{\partial z}{\partial y}\left(\frac{\partial}{\partial x}\right)_{y}\left(\frac{F_{x}}{\partial x}\right)_{y}\left(\frac{F_{x}}{F_{z}}\right)-\frac{F_{x z} F_{z} \frac{\partial z}{\partial x}-F_{z z} F_{x} \frac{\partial z}{\partial x}}{F_{z}^{2}}\right\} \\
& =\frac{\partial z}{\partial y} \frac{\partial^{2} z}{\partial x^{2}}-\frac{\partial z}{\partial x} \frac{\partial^{2} z}{\partial y \partial x} \cdot
\end{aligned}
$$

